\title{
Single Amino Acid Mutation around Flavin Cofactor Changes pH-Dependence of Basidiomycetes Class I Cellobiose Dehydrogenase Activity
}

\author{
(Received November 27, 2012; Accepted December 17, 2012) \\ (J-STAGE Advance Published Date: January 30, 2013) \\ Kiyohiko Igarashi $^{1, *}$ and Masahiro Samejima ${ }^{1}$ \\ ${ }^{1}$ Department of Biomaterial Sciences, Graduate School of Agricultural and Life Sciences, The University of Tokyo \\ (1-1-1 Yayoi, Bunkyo-ku, Tokyo 113-8657, Japan)
}

\begin{abstract}
Fungal cellobiose dehydrogenases (CDHs) are divided on the basis of amino acid sequence into class I (from basidiomycetes) and class II (from ascomycetes), which show quite different pH-dependence of the activity. Here, we mutated glutamine 734 (Q734) in the flavin domain of class I CDH from the basidiomycete Phanerochaete chrysosporium to the corresponding amino acid in class II CDHs (serine or threonine), and compared the kinetics of the mutant enzymes (Q734S and Q734T) and wild-type enzyme (WT). The two mutant enzymes showed almost identical absorption spectra, although that of WT was slightly different. When the steady-state kinetic parameters were compared at pH 4.0 and 7.0, WT showed the highest activity at both $\mathrm{pH}$ values. However, $k \mathrm{cat} / K \mathrm{~m}$ for Q734S was similar at both $\mathrm{pHs}$, whereas $k$ cat/ $K \mathrm{~m}$ for Q734T was 3.5 times higher at $\mathrm{pH} 4.0$ than that at $\mathrm{pH}$ 7.0. As for the $\mathrm{pH}$-dependence of the specific activity, Q734S did not have an apparent optimum $\mathrm{pH}$ in the $\mathrm{pH}$ range tested, whereas Q734T showed an acidic optimum pH profile compared with WT. These differences can be explained in terms of the effect of the side chain of the amino acid residue at position 734 on the reactivity of the flavin cofactor.
\end{abstract}

Key words: cellobiose dehydrogenase, Phanerochaete chrysosporium, flavin adenine dinucleotide, Humicola insolens

\section{INTRODUCTION}

Cellobiose dehydrogenase $(\mathrm{CDH}, \mathrm{EC} 1.1 .99 .18)$ is an extracellular flavocytochrome produced by cellulolytic fungi. It catalyzes oxidation of the reducing-end of cellobiose and cellooligosaccharides (hydrolytic products of cellulose) and produces the corresponding $\delta$-lactones, using various quinones and ferric compounds as electron acceptors. ${ }^{1-3)}$ This enzyme was initially isolated from the wood-rotting basidiomycete Phanerochaete chrysosporium as two different proteins, i.e., cellobiose oxidase (CBO, EC 1.1.3.25), which is a flavocytochrome oxidizing cellobiose with molecular oxygen as an electron acceptor, ${ }^{4}$ ) and cellobiose:quinone oxidoreductase (CBQ, EC 1.1.5.1), which is a flavo-protein utilizing quinones as electron acceptors. ${ }^{5,6)}$ These enzymes were thought to be interface enzymes between cellulose and lignin degradation because of their substrate specificity for cellulolytic and lignolytic products as the electron donor and acceptor, respectively. However, later reports established that $\mathrm{CBQ}$ is a proteolytic product of $\mathrm{CBO},{ }^{7)} \mathrm{CBO}$ utilizes oxygen more slowly than other redox compounds, ${ }^{8-10)}$ and $\mathrm{CDH}$ is present in many other non-lignolytic cellulolytic fungi. ${ }^{1{ }^{1-13)}}$ Therefore, CBO and $\mathrm{CBQ}$ are now both categorized as $\mathrm{CDH}$, and the physio-

\footnotetext{
*Corresponding author (Tel. +81-3-5841-5258, Fax. +81-3-5841-5273, E-mail: aquarius@mail.ecc.u-tokyo.ac.jp).

Abbreviations: CBO, cellobiose oxidase; $\mathrm{CBQ}$, cellobiose: quinone oxidoreductase; $\mathrm{CDH}$, cellobiose dehydrogenase; FAD, flavin adenine dinucleotide; GMC, glucose-methanol-choline; Hi, Humicola insolens; Pc, Phanerochaete chrysosporium; WT, wild-type.
}

logical function of the enzyme has been characterized primarily as cellulose degradation in recent decades. ${ }^{14,15)}$

$\mathrm{CDH}$ consists of two major domains, i.e., the flavin- and heme-containing domains. ${ }^{4,16,17)}$ The flavin chromophore in $\mathrm{CDH}$ is $\mathrm{FAD}$, which is responsible for the oxidation of cellobiose. ${ }^{10,16,18)}$ The flavin domain belongs to the glucosemethanol-choline (GMC) oxidoreductase family, ${ }^{19,20)}$ although the heme domain is unique to $\mathrm{CDH}^{21,22)}$ Some CDHs from ascomycetes carry a family 1 carbohydratebinding module in addition to the flavin and cytochrome domains. ${ }^{23)}$ A recent phylogenetic analysis of the amino acid sequence of the flavin domain indicated that CDHs can be divided into class I (from basidiomycetes) and class II (from ascomycetes), together with class III, which contains putative ascomycetes gene products. ${ }^{24,25)}$ The basiomycetes $\mathrm{CDH}$ commonly exhibits acidic pH-dependence of cytochrome $c$ reduction, whereas reduction of quinone-like electron acceptors is flavin-dependent and shows a rather broad $\mathrm{pH}$ dependence. ${ }^{10,26,27)}$ However, the $\mathrm{pH}$ dependence of ascomycetes class II CDH is species-dependent, and no clear pattern has been found in this class. ${ }^{25}$

We previously compared the kinetic characteristics of classes I and II CDHs from P. chrysosporium (PcCDH) and the ascomycete Humicola insolens $(\mathrm{HiCDH})$, respectively, and showed that the difference in $\mathrm{pH}$ dependence of the two enzymes is caused by the $\mathrm{pH}$-dependent reactivity of the flavin domain isolated after proteolysis of the intact enzymes containing both flavin and cytochrome domains. ${ }^{26)} \mathrm{We}$ speculated that the different reactivity of flavin domains from classes I and II CDHs arises partly because of the 
involvement of modified flavin, 6-hydroxyl-FAD, as an active cofactor in $\mathrm{HiCDH}$. However, several other class II CDHs have since been cloned from ascomycetes and characterized, and the $\mathrm{pH}$-dependence of these enzymes also differs from that of class I CDH despite the absence of cofactor modification, suggesting that the difference arises from a difference of protein structure. ${ }^{25)}$

In the present study, we predicted the structure of the flavin domain of $\mathrm{HiCDH}$ based on the three-dimensional structure of the flavin domain of $P c \mathrm{CDH}$, and found that glutamine 734 (Q734) of $P c \mathrm{CDH}$, among amino acid residues around the flavin, is mutated to serine in $\mathrm{HiCDH}$. Based on alignment of the amino acid sequences of class II CDHs, we designed two types of mutated PcCDH (Q734S and Q734T) and investigated their reactivity, especially its $\mathrm{pH}$ dependence, in order to understand the structural basis for the difference of reactivity between classes I and II CDHs.

\section{MATERIALS AND METHODS}

Materials. The gene coding cellobiose dehydrogenase $(c d h)$, previously cloned from Phanerochaete chrysosporium strain K-3 into pPIC9K vector (pPIC9K/cdh), was used as a template. ${ }^{28)}$ Escherichia coli strain JM109 (Takara Bio Inc., Ohtsu, Japan) and Pichia pastoris strain KM71H (Invitrogen Co., Ltd., Carlsbad, USA) were used as the subcloning host and for heterologous production of the recombinant $\mathrm{CDH}$, respectively. D-Cellobiose was purchased from ICN Biomedicals, Inc. (Irvine, USA), ubiquinone (2,3-dimethoxy-5-methyl-1,4-benzoquinone) was purchased from Wako Pure Chemical Industries, Ltd. (Osaka, Japan), and flavin adenine dinucleotide (FAD) was purchased from Sigma Chemical Co. (St Louis, USA). To assess the $\mathrm{pH}$ dependence, $50 \mathrm{mM}$ buffers were used as described previously. ${ }^{26,27)}$

Prediction of three-dimensional structure of the flavin domain of $\mathbf{H i C D H}$. The three-dimensional structure of $\mathrm{HiCDH}$ was predicted using the Phyre $^{2}$ Protein Fold Recognition Server (http://www.sbg.bio.ic.ac.uk/phyre2/ index.cgi). ${ }^{29)}$ The amino acid sequence of the flavin domain of $\mathrm{HiCDH}$ was input into the server and the model structure was constructed using the "intensive" mode of the server. The PDB file constructed by the server was visualized with MacPymol (Ver. 1.3, Schrodinger, LLC), and the model structure was compared with the crystal structure of the flavin domain of PcCDH (PDB: 1NAA). ${ }^{30)}$ Based on this structural comparison and amino acid sequence alignment with other fungal CDHs using ClustalW2 (http://www.ebi. ac.uk/Tools/msa/clustalw2/), three mutants (Q734H, Q734S and Q734T) were designed for further experiments.

Preparation of wild-type and mutant flavin domains. Recombinant wild-type (WT) and mutant flavin domains from $P$. chrysosporium $\mathrm{CDH}$ were heterologously expressed in the methylotropic yeast $P$. pastoris and purified based on the procedures used in previous reports. ${ }^{28,31-33)}$ The gene coding the flavin domain of $\mathrm{PcCDH}$ was subcloned into pPICZ $\alpha$-A vector from $\mathrm{pPIC9K} / c d h$ using the following primers:

PcFAD-EcoRI-F: 5'-TTTGAATTCACACCTTACGATTACATC-3';
PcFAD-XbaI-R: $\quad$ 5'-TTTTCTAGATTAAGGACCTCCCGCAAG-3'

(restriction enzyme sites are shown in italic). The fragment was subcloned into the $\mathrm{pCR}^{\circledR} 4 \mathrm{Blunt}-\mathrm{TOPO}$ vector (Invitrogen Co., Ltd.) according to the manufacturer's instructions. The vector pCR $4{ }^{\circledR}$ Blunt-TOPO/flavin was digested with EcoRI and $X b a \mathrm{I}$ (Takara Bio Inc.) after miniprep and ligated into the pPICZ $\alpha$-A vector (Invitrogen Co., Ltd.) at the same restriction sites. Site-directed mutagenesis was carried out by $D p n I$ restriction enzyme treatment after inverse PCR as described elsewhere. ${ }^{34)}$ The following synthetic oligonucleotides:

Q734H-F： 5'-CCCACGGGCAACCCCCACGGCACGCTCATGTCT-3';

Q734H-R: 5'-AGACATGAGCGTGCCGTGGGGGTTGCCCGTGGG -3'

Q734S-F: 5'-CCCACGGGCAACCCCTCAGGCACGCTCATGTCT-3';

Q734S-R: 5'-AGACATGAGCGTGCCTGAGGGGTTGCCCGTGGG-3';

Q734T-F: 5'-CCCACGGGCAACCCCACGGGCACGCTCATGTCT-3';

Q734T-R: 5'-AGACATGAGCGTGCCCGTGGGGTTGCCCGTGGG $-3^{\prime}$

(mutated nucleotides are underlined) and KOD-Plus (version 2; Toyobo Co., Ltd., Osaka) were used to obtain pPICZ $\alpha$ / $q 734 h, \mathrm{pPICZ} \alpha / q 734 s$ and $\mathrm{pPICZ} \alpha / q 734 t$ according to the manufacturer's instructions.

After miniprep of all constructs, approximately $3 \mu \mathrm{g}$ of plasmid was linearized with Bpu1102I (TaKaRa Bio, Inc., Shiga, Japan) and transformed into Pichia pastoris KM-71H using a MicroPulser electroporation device (Bio-Rad Laboratories, Inc., Hercules, USA). The zeocin-resistant transformant was cultivated in growth medium $(1 \%$ yeast extract, $2 \%$ polypeptone and $1 \%$ glycerol) for $24 \mathrm{~h}$ at $30^{\circ} \mathrm{C}$, then cultivated in induction medium ( $1 \%$ yeast extract, $2 \%$ polypeptone and $1 \%$ methanol) for $48 \mathrm{~h}$ at $26.5^{\circ} \mathrm{C}$, and the recombinant enzymes were purified from the culture filtrate using the same protocol as described previously. ${ }^{26,28}$ The purity and concentration of the enzymes were confirmed by SDS-PAGE and by measurement of the absorption spectrum with a UV/Vis spectrophotometer (UV-1600PC, Shimadzu Corporation, Tokyo, Japan).

Steady-state enzyme assays. To obtain the steady-state kinetic parameters of cellobiose oxidation, the reduction rate of $1 \mathrm{mM}$ ubiquinone was plotted versus concentration of cellobiose ( 0 to $3 \mathrm{mM}$ ) with $100 \mathrm{nM}$ enzyme in $50 \mathrm{mM}$ buffer of various $\mathrm{pH}$ values, as described previously. ${ }^{26,27)}$ The reduction of ubiquinone was monitored photometrically at $406 \mathrm{~nm}\left(\Delta \varepsilon_{406}=0.745 \mathrm{mM}^{-1} \mathrm{~cm}^{-1}\right)$. Since apparent substrate inhibition was observed, the obtained substrate dependence plots were fitted to the Michaelis-Menten equation with a substrate inhibition constant $(K \mathrm{i})$ as described previously. ${ }^{27)}$ Steady-state kinetic parameters $(K \mathrm{~m}, k$ cat and $K$ i) were estimated by non-linear fitting of the data using DeltaGraph (Ver. 6.0, Red Rock Software, Inc., Salt Lake City, USA) and KaleidaGraph (Ver. 4.0, Synergy Software, Reading, USA). 


\section{RESULTS AND DISCUSSION}

$\mathrm{CDH}$ is the only flavocytochrome containing flavin and $b$-type cytochrome that is known to be secreted extracellularly during the course of fungal cellulose degradation. Its physiological function is still unclear. Quinone reduction is known to be catalyzed mainly by flavin, whereas electron transfer via the cytochrome domain is necessary to reduce ferric compounds such as cytochrome $c .{ }^{9,10)}$ Therefore, the $\mathrm{pH}$-dependence of the enzyme activity is quite complex, depending on the electron acceptor, i.e., quinone reduction mainly reflects the velocity of flavin-dependent cellobiose oxidation, whereas cytochrome $c$ reduction is much more affected by the electron transfer rate from flavin to $b$-type cytochrome, as reported previously. ${ }^{18,27,32)}$ Although we focused on electron transfer between the two domains in our previous studies, ${ }^{27,32)}$ the effect of the flavin environment on the reactivity of this enzyme, especially the $\mathrm{pH}$-dependence, is also important in order to fully understand the reaction of $\mathrm{CDH}$. In the present study, therefore, several mutants were designed with amino acid substitutions around the flavin cofactor, and the $\mathrm{pH}$ profiles of these mutants were investi- gated in comparison with that of WT.

A comparison of the crystal structure of the flavin domain of $P c \mathrm{CDH}$ and the model structure of the $\mathrm{HiCDH}$ flavin domain predicted by the Phyre ${ }^{2}$ server indicated only a single amino acid difference around the flavin, i.e., ${ }^{734} \mathrm{Gln}$ (Q734) in $P_{c} \mathrm{CDH}$ corresponds to ${ }^{744} \mathrm{Ser}$ in $\mathrm{HiCDH}$, as shown in Figs. $1 \mathrm{~A}$ and B. Based on sequence alignment with fungal $\mathrm{CDH}$, the corresponding residue is Gln or His in basidiomycetes class I CDHs, whereas it is Ser or Thr in ascomycetes class II CDHs, as shown in Fig. 1C. Therefore, we designed three constructs for the recombinant flavin domain of PcCDH (Q734H, Q734S and Q734T), and P. pastoris was transformed using the obtained plasmids. Two mutants (Q734S and Q734T), as well as WT were successfully produced, although Q734H could not be obtained in several trials using different batches of construct, possibly because the mutation induced incorrect folding. Therefore, we used only the former three flavin domains in the following experiments.

WT, Q734S and Q734T were heterologously expressed in $P$. pastoris and were purified via three steps of column chromatography (hydrophobic interaction, anion-exchange

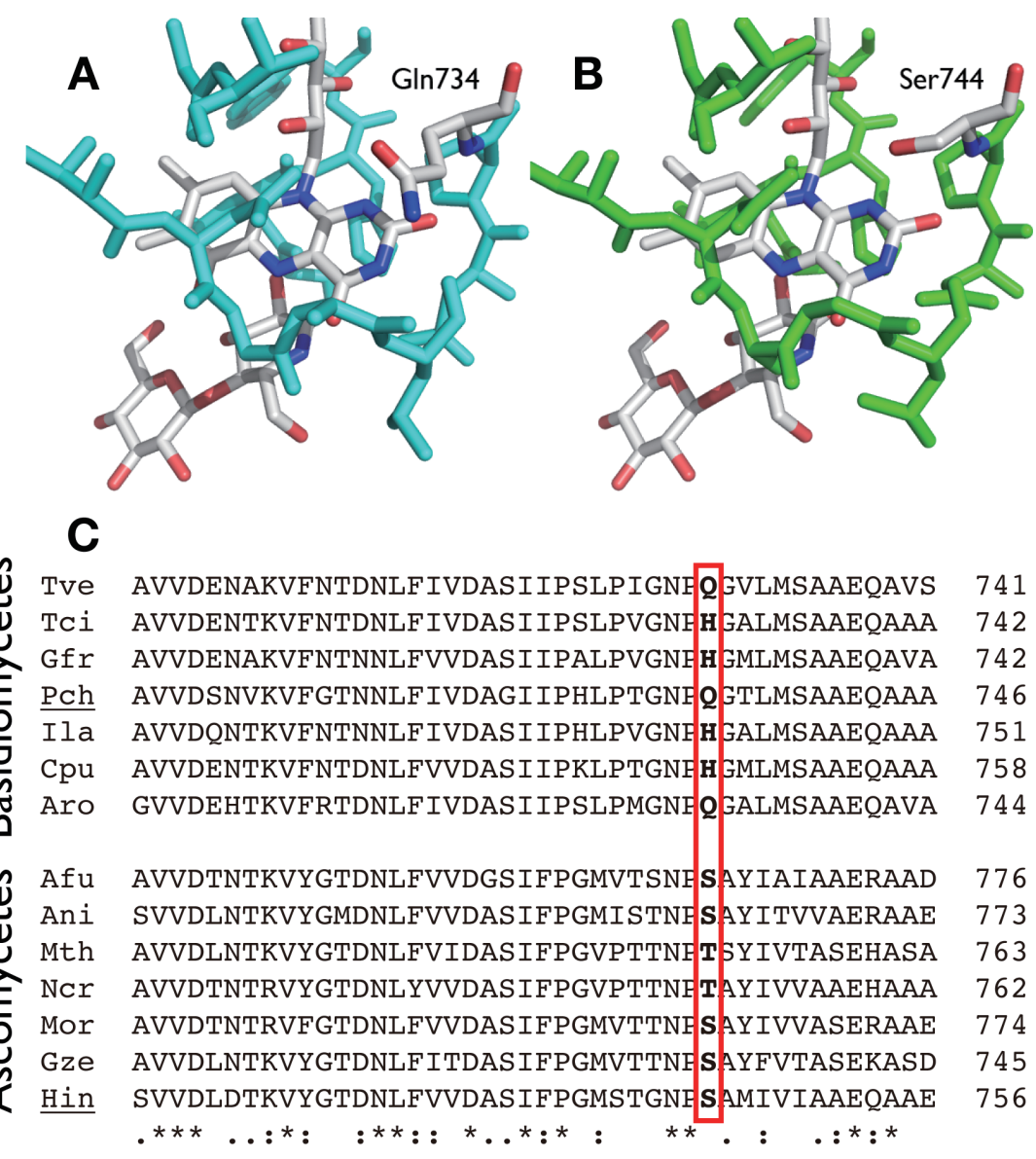

Fig. 1. Amino acids around the flavin cofactor in $P c \mathrm{CDH}(\mathrm{A})$, those in the predicted structure of $\mathrm{HiCDH}(\mathrm{B})$, amino acid sequence alignment of $\mathrm{CDHs}$ from basidiomycetes and ascomycetes $(\mathrm{C})$.

The amino acid sequences of $\mathrm{CDHs}$ used for the alignments are those of Trametes versicolor (Tve, AAC50004), T. cinnabarina (Tci, AAC32197), Grifola frondosa (Gfr, BAC20641), P. chrysosporium (Pch, AAB92262), Irpex lacteus (Ila, BAD36748), Coniophora puteana (Cpu, BAD32781), Athelia rolfsii (Aro, AAO64483), Aspergillus fumigatus (Afu, EDP55266), A. nidulans (Ani, XP_680499), Myceliophthora thermophila (Mth, XP_003663382), Neurospora crassa (Ncr, XP_956591), Magnaporthe oryzae (Mor, XP 003712902), Gibberella zeae (Gze, XP 389261) and H. insolens (Hin, AAF69005). The amino acids corresponding to glutamine 734 (Q734) in P. chrysosporium $\mathrm{CDH}$ are boxed. 
and gel filtration). The adsorption spectra of the purified flavin domains are shown in Figs. 2A-C. All three proteins showed spectra with peaks at approximately 275,385 and $460 \mathrm{~nm}$, suggesting the presence of a flavin chromophore, and the $A 460 / A 280$ values of WT, Q734S and Q734T were $0.086,0.084$ and 0.085 , respectively, indicating that the flavin content is almost the same in the three protein preparations. Moreover, a comparison with the absorption spectrum of proteolytically isolated flavin domain from intact $P c \mathrm{CDH}^{10,16)}$ indicated that most of the protein molecules contain flavin cofactor. When the spectra were analyzed in detail, however, there were differences between WT and the mutants, especially at around $485 \mathrm{~nm}$. WT has a small shoulder at $485 \mathrm{~nm}$, which is also found in the flavin domain isolated from native $P c \mathrm{CDH}$ purified from cellulolytic culture of $P$. chrysosporium. In contrast, neither Q734S and Q734T showed the shoulder at $485 \mathrm{~nm}$, and the spectra were similar to that of free FAD, although the peak top is shifted owing to the influence of the surrounding protein. In flavoprotein, the shoulder has been shown to reflect the environment of the flavin cofactor. ${ }^{35)}$ Therefore, this small difference between WT and mutant enzymes could be an indicator of the relative reactivity of the flavin.

As shown in Fig. 3, steady-state kinetic experiments were carried out at $\mathrm{pH} 4.0$ and 7.0 using various concentrations of cellobiose as a substrate. The course of the reaction was monitored in terms of ubiquinone reduction. The kinetic parameters, $K \mathrm{~m}, k_{\mathrm{cat}}$, $k_{\mathrm{cat}} / K_{\mathrm{m}}$ and $K \mathrm{i}$, obtained are listed in Table 1. In all cases, the cellobiose concentration dependence of cellobiose oxidation showed substrate inhibition, and the activity decreased with increase of the substrate concentration. This is mainly because cellobiose oxidation and electron transfer to the electron acceptor are carried out at the same site, as described previously. ${ }^{27)}$ At both $\mathrm{pH}$ values, WT had the highest reactivity among the three enzymes, though the activity was higher at $\mathrm{pH} 4.0$ than at $\mathrm{pH} 7.0$. Interestingly, Q734T has higher activity than Q734S at $\mathrm{pH}$ 4.0, whereas Q734S is much more active than Q734T at $\mathrm{pH}$ 7.0. We found that the kinetic efficiency $(k$ cat $/ K \mathrm{~m})$ at $\mathrm{pH} 4.0$ was 2.8 and 3.5 times higher than that at $\mathrm{pH} 7.0$ for WT and
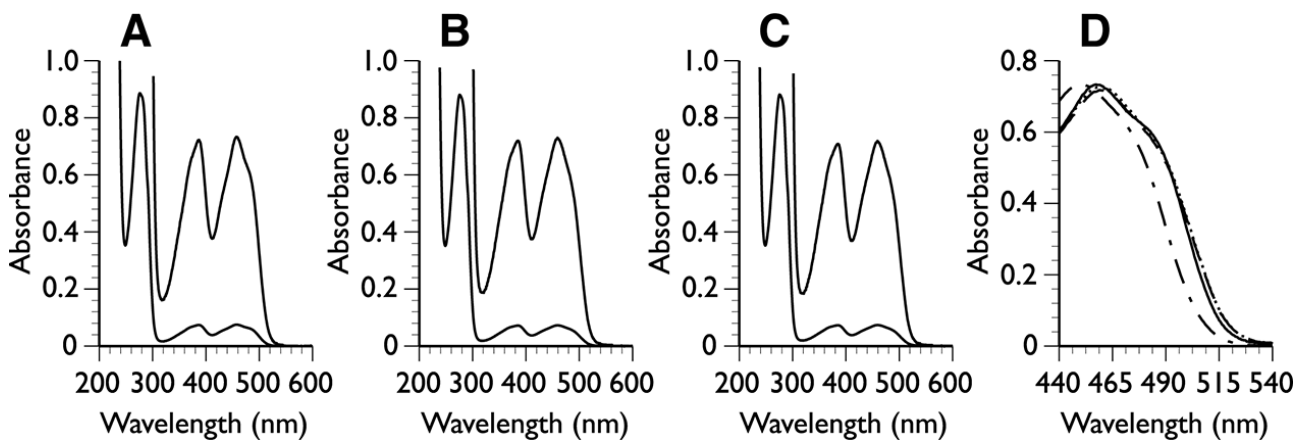

Fig. 2. Absorption spectra of WT (A), Q734S (B), Q734T (C) and comparison of the spectra of these flavin domains and free FAD (D).

The spectra of purified flavin domains and $\operatorname{FAD}(6.5 \mu \mathrm{M})$ were monitored as described in the Materials and Methods section. The upper spectra in $\mathrm{A}-\mathrm{C}$ are 10 times magnified versions of those originally obtained. The solid, dotted, dashed, and dot-dashed lines in D indicate WT, Q734S, Q734T and free FAD, respectively.
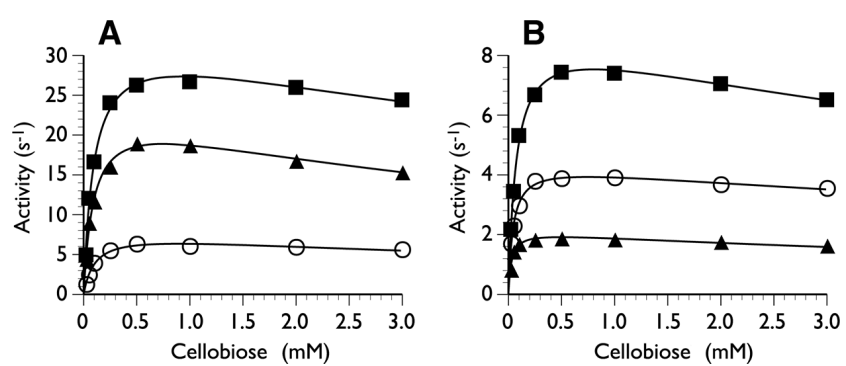

Fig. 3. Cellobiose concentration dependence of ubiquinone-reducing activity by WT (solid square), Q734S (open circle) and Q734T (solid triangle).

Various concentrations of cellobiose $(0-3 \mathrm{mM})$ were incubated with $1 \mathrm{mM}$ ubiquinone in $50 \mathrm{mM}$ sodium acetate buffer, $\mathrm{pH} 4.0$ (A) and 50 mM HEPES buffer, pH 7.0 (B), and the activity was estimated from the decrease of absorption at $406 \mathrm{~nm}$.
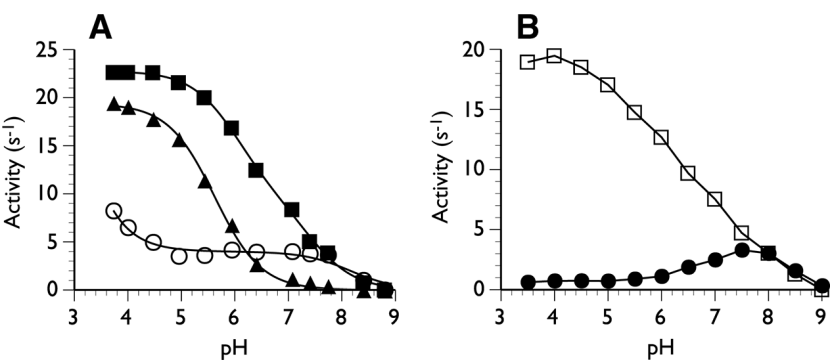

Fig. 4. $\mathrm{pH}$ dependence of ubiquinone-reducing activity by WT (solid square), Q734S (open circle) and Q734T (solid triangle) (A) and those of the flavin domain isolated from $\mathrm{PcCDH}$ (open square) and $\mathrm{HiCDH}$ (solid circle) (B).

The activity was estimated in a mixture of $1 \mathrm{mM}$ cellobiose and $1 \mathrm{mM}$ ubiquinone in various $50 \mathrm{mM}$ buffers.

Table 1. Steady-state kinetic parameters of cellobiose oxidation by WT and mutants using ubiquinone as an electron acceptor.

\begin{tabular}{|c|c|c|c|c|c|c|c|c|}
\hline & \multicolumn{4}{|c|}{$\mathrm{pH} 4.0$} & \multicolumn{4}{|c|}{$\mathrm{pH} 7.0$} \\
\hline & $\begin{array}{c}K \mathrm{~m} \\
(\mu \mathrm{M})\end{array}$ & $\begin{array}{l}k \text { cat } \\
\left(\mathrm{s}^{-1}\right)\end{array}$ & $\begin{array}{c}k \mathrm{cat} / K \mathrm{~m} \\
\left(\mathrm{~s}^{-1} \cdot \mu \mathrm{M}^{-1}\right)\end{array}$ & $\begin{array}{c}K \mathrm{i} \\
(\mathrm{mM})\end{array}$ & $\begin{array}{c}K \mathrm{~m} \\
(\mu \mathrm{M})\end{array}$ & $\begin{array}{l}k \text { cat } \\
\left(\mathrm{s}^{-1}\right)\end{array}$ & $\begin{array}{c}k \mathrm{cat} / K \mathrm{~m} \\
\left(\mathrm{~s}^{-1} \cdot \mu \mathrm{M}^{-1}\right)\end{array}$ & $\begin{array}{c}K \mathrm{i} \\
(\mathrm{mM})\end{array}$ \\
\hline WT & $97.4 \pm 13.6$ & $33.0 \pm 1.7$ & 0.34 & $9.00 \pm 2.62$ & $74.2 \pm 4.8$ & $8.93 \pm 0.20$ & 0.12 & $8.59 \pm 1.11$ \\
\hline Q734S & $106 \pm 14$ & $7.85 \pm 0.40$ & 0.074 & $7.60 \pm 1.94$ & $43.1 \pm 3.3$ & $4.38 \pm 0.10$ & 0.10 & $12.8 \pm 2.4$ \\
\hline Q734T & $94.4 \pm 10.8$ & $23.6 \pm 1.0$ & 0.25 & $5.89 \pm 1.10$ & $29.6 \pm 5.2$ & $2.13 \pm 0.10$ & 0.072 & $10.2 \pm 3.3$ \\
\hline
\end{tabular}

Various concentrations of cellobiose $(0-3 \mathrm{mM})$ were oxidized by $100 \mathrm{nM}$ flavin domain with monitoring of ubiquinone reduction as described in the Materials and Methods section. These parameters were calculated from non-linear curve fitting of Fig. 3. 

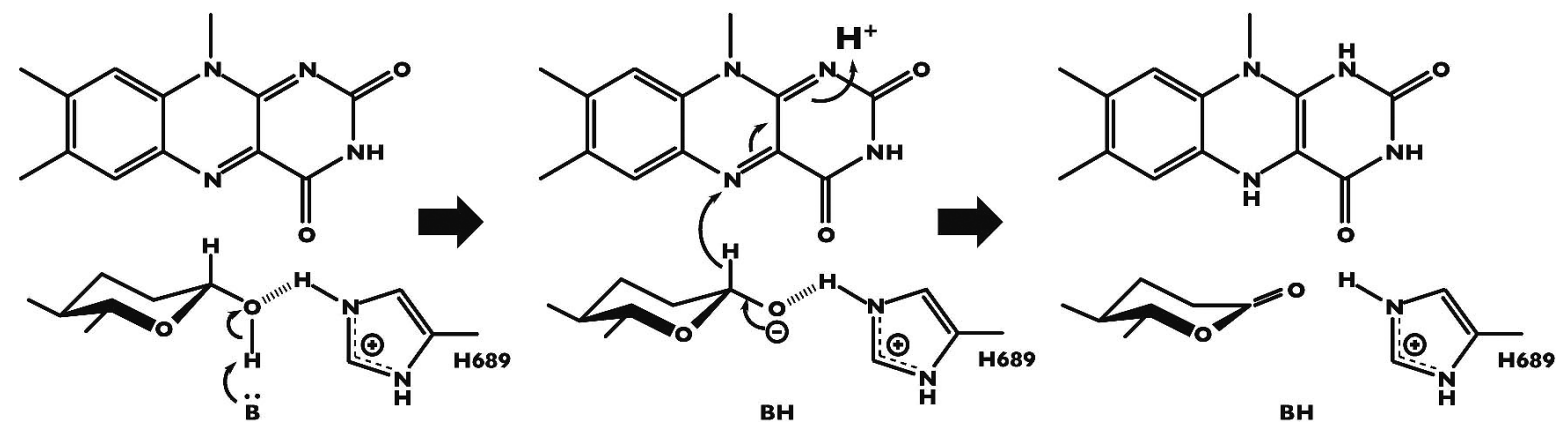

Fig. 5. Predicted reaction mechanisms of flavin cofactor in $P c \mathrm{CDH}$.

The proton at the N1 position is shown in the middle of this figure. Histidine 689 (H689) is the catalytic residue and B (Asn732) is a possible basic residue.

Q734T, respectively, whereas the value for Q734S was 0.73 at both pHs. This clearly indicates that a single amino acid mutation around the flavin cofactor changes the $\mathrm{pH}$-dependence of the activity without significantly decreasing the kinetic efficiency.

The $\mathrm{pH}$-dependence of ubiquinone reduction was compared among WT, Q734S and Q734T (Fig. 4A). The pH profile of Q734S was completely different from those of the other two enzymes. Q734S showed quite similar activity to WT at alkaline $\mathrm{pH}$ (more than 7.5), whereas the activity did not increase with decrease of $\mathrm{pH}$, as it did in the cases of WT and Q734T. Thus, the activity of Q743S was almost $\mathrm{pH}$-independent in the acidic $\mathrm{pH}$ range examined. In contrast, the $\mathrm{pH}$ profile of Q734T has a rather acidic $\mathrm{pH}$ optimum compared with that of WT. The activity of the mutant decreases dramatically with increase of $\mathrm{pH}$ and falls to a half of the maximum activity at $\mathrm{pH} 5.5$, which is lower than that in the case of WT ( $\mathrm{pH}$ 6.5). These results indicated that the mutation of Gln734 to Ser causes inactivation at acidic $\mathrm{pH}$, whereas the mutation to Thr inactivates the flavin at alkaline $\mathrm{pH}$ in basidiomycetes class I CDH. Fig. 4B shows the $\mathrm{pH}$-dependence of quinone reduction by the flavin domain proteolytically isolated from native $\mathrm{CDH}$ produced by $P$. chrysosporium and $\mathrm{H}$. insolens. ${ }^{26)} \mathrm{HiCDH}$ is an alkalophilic enzyme, like many of the cellulolytic enzymes produced by the fungus. However, if the actual activity of the flavin domain is compared with that in the case of $\mathrm{PcCDH}$, the $\mathrm{pH}$ profile of the flavin domain of $\mathrm{HiCDH}$ shows a similar tendency to that of Q734S, and it is not "alkalophilic", but rather "inactive at acidic pH", as shown in Fig. 4B. Including our previous report, there are several reports not only of different $\mathrm{pH}$ profiles between basidiomycetes class I and ascomycetes class II CDHs, but also of different $\mathrm{pH}$ profiles within class II CDHs. However, as shown in the present study, it appears that the activity is highly dependent on the environment of the flavin cofactor, and even a single amino acid mutation is enough to change the $\mathrm{pH}$ profile of $\mathrm{CDH}$ activity. It was unexpected that the Ser and Thr mutants showed markedly different $\mathrm{pH}$ profiles. Ser and Thr are usually considered to be very similar, because both have hydrophilic character, and so it was expected that Q734S and Q734T would show similar behavior. Although the $\mathrm{pH}$ dependence of Q734S did not completely mimic that of the flavin domain of $\mathrm{HiCDH}$, other factors seem to affect the reactivity of flavin.

It is possible to interpret the effects of mutation at this position in terms of the mechanism of cellobiose dehydroge- nation catalyzed by $\mathrm{CDH}$. As shown in Fig. 5, the existence of a proton around $\mathrm{N} 1$ of the isoaloxazine ring is important for substrate dehydrogenation to proceed, and the preference for cellobiose oxidation at acidic $\mathrm{pH}$ by $\mathrm{CDH}$ has been explained in terms of the presence of this proton. However, in the Q734S mutant, the proton would hardly be involved because of the hydroxyl group of the Ser residue. Since the acidic $\mathrm{pH}$ profile was partly recovered in Q734T, the methyl group of the Thr residue might favor protonation and/or change the conformation of the hydroxyl group so that it does not interact with $\mathrm{N} 1$ of flavin. There is another possibility that the redox potential of the flavin cofactor is decreased in Q734S, i.e., hydroxyl group of Ser residue lowers the redox potential of the cofactor. In $H$. insolens $\mathrm{CDH}$, we found the modified flavin with hydroxylation at C6 position, and showed that the reactivity of the cofactor is decreased by the modification. ${ }^{26)}$ Nevertheless, a detailed explanation must await structure determination of Q734S and/or the flavin domain of $\mathrm{HiCDH}$. Moreover, since we were unable to produce a His mutant at the Gln734 position, we cannot discuss the full range of activity variation in naturally occurring CDHs. Further biochemical and molecular biological studies will be necessary to understand in detail the pH-dependence of classes I and II CDHs.

\section{ACKNOWLEDGMENTS}

This research was supported by a grant from the Advanced Low Carbon Technology Research and Development Program (ALCA) of the Japan Science and Technology Agency (JST) to K.I., by Grants-inAid for Scientific Research for Young Scientists (A, No. 19688016 and 21688023) and (B, No. 17780245), by a Grant-in-Aid for Scientific Research (B, No. 24380089), and by a Grant-in-Aid for Innovative Areas (No. 24114008) to K.I. from the Japanese Ministry of Education, Culture, Sports, and Technology (MEXT).

\section{REFERENCES}

1 ) G. Henriksson, G. Johansson and G. Pettersson: A critical review of cellobiose dehydrogenases. J. Biotechnol., 78, 93-113 (2000).

2 ) M. Zamocky, R. Ludwig, C. Peterbauer, B.M. Hållberg, C. Divne, P. Nicholls and D. Haltrich: Cellobiose dehydrogenase: a flavocytochrome from wood-degrading, phytopathogenic and saprotropic fungi. Curr. Prot. Pept. Sci., 7, 255-280 (2006).

3 ) K.E.L. Eriksson, N. Habu and M. Samejima: Recent advances in fungal cellobiose oxidoreductases. Enzyme Microb. Technol., 15, 1002-1008 (1993).

4 ) A.R. Ayers, S.B. Ayers and K.E. Eriksson: Cellobiose oxidase, purification and partial characterization of a hemoprotein from Sporotrichum pulverulentum. Eur. J. Biochem., 90, 171-181 (1978). 
5 ) U. Westermark and K.E. Eriksson: Cellobiose-quinone oxidoreductase, a new wood-degrading enzyme from white-rot fungi. Acta Chem. Scand. B, 28, 209-214 (1974).

6 ) U. Westermark and K.E. Eriksson: Purification and properties of cellobiose-quinone oxidoreductase from Sporotrichum pulverulentum. Acta Chem. Scand. B, 29, 419-424 (1975).

7 ) J.D. Wood and P.M. Wood: Evidence that cellobiose:quinone oxidoreductase from Phanerochaete chrysosporium is a breakdown product of cellobiose oxidase. Biochim. Biophys. Acta, 1119, 90-96 (1992).

8 ) M.T. Wilson, N. Hogg and G.D. Jones: Reactions of reduced cellobiose oxidase with oxygen: Is cellobiose oxidase primarily an oxidase? Biochem. J., 270, 265-267 (1990).

9 ) S.M. Kremer and P.M. Wood: Evidence that cellobiose oxidase from Phanerochaete chrysosporium is primarily an $\mathrm{Fe}(\mathrm{III})$ reductase. Kinetic comparison with neutrophil NADPH oxidase and yeast flavocytochrome b2. Eur. J. Biochem., 205, 133-138 (1992).

10) M. Samejima and K.E. Eriksson: A comparison of the catalytic properties of cellobiose:quinone oxidoreductase and cellobiose oxidase from Phanerochaete chrysosporium. Eur. J. Biochem., 207, 103-107 (1992).

11) M.R. Coudray, G. Canevascini and H. Meier: Characterization of a cellobiose dehydrogenase in the cellulolytic fungus Sporotrichum (Chrysosporium) thermophile. Biochem. J., 203, 277-284 (1982).

12) J.C. Sadana and R.V. Patil: The purification and properties of cellobiose dehydrogenase from Sclerotium rolfsii and its role in cellulolysis. J. Gen. Microbiol., 131, 1917-1923 (1985).

13) C. Schou, M.H. Christensen and M. Schülein: Characterization of a cellobiose dehydrogenase from Humicola insolens. Biochem. J., 330, 565-571 (1998).

14) W.J. Bao and V. Renganathan: Cellobiose oxidase of Phanerochaete chrysosporium enhances crystalline cellulose degradation by cellulases. FEBS Lett., 302, 77-80 (1992).

15) K. Igarashi, M. Samejima and K.E. Eriksson: Cellobiose dehydrogenase enhances Phanerochaete chrysosporium cellobiohydrolase I activity by relieving product inhibition. Eur. J. Biochem., 253, 101-106 (1998).

16) F.F. Morpeth: Some properties of cellobiose oxidase from the white-rot fungus Sporotrichum pulverulentum. Biochem. J., 228, 557-564 (1985).

17) W.J. Bao, S.N. Usha and V. Renganathan: Purification and characterization of cellobiose dehydrogenase, a novel extracellular hemoflavoenzyme from the white-rot fungus Phanerochaete chrysosporium. Arch. Biochem. Biophys., 300, 705-713 (1993).

18) M. Samejima, R.S. Phillips and K.E. Eriksson: Cellobiose oxidase from Phanerochaete chrysosporium. Stopped-flow spectrophotometric analysis of $\mathrm{pH}$-dependent reduction. FEBS Lett., 306, 165-168 (1992).

19) M. Raices, E. Paifer, J. Cremata, R. Montesino, J. Stahlberg, C. Divne, I.J. Szabo, G. Henriksson, G. Johansson and G. Pettersson: Cloning and characterization of a cDNA encoding a cellobiose dehydrogenase from the white rot fungus Phanerochaete chrysosporium. FEBS Lett., 369, 233-238 (1995).

20) M. Zamocky, M. Hållberg, R. Ludwig, C. Divne and D. Haltrich: Ancestral gene fusion in cellobiose dehydrogenases reflects a specific evolution of GMC oxidoreductases in fungi. Gene, $\mathbf{3 3 8}$, 1-14 (2004).

21) M.C. Cox, M.S. Rogers, M. Cheesman, G.D. Jones, A.J. Thomson, M.T. Wilson and G.R. Moore: Spectroscopic identifi- cation of the heme ligands of cellobiose oxidase. FEBS Lett., $\mathbf{3 0 7}$, 233-236 (1992).

22) B.M. Hållberg, T. Bergfors, K. Backbro, G. Pettersson, G. Henriksson and C. Divne: A new scaffold for binding haem in the cytochrome domain of the extracellular flavocytochrome cellobiose dehydrogenase. Structure, 8, 79-88 (2000).

23) S.S. Subramaniam, S.R. Nagalla and V. Renganathan: Cloning and characterization of a thermostable cellobiose dehydrogenase from Sporotrichum thermophile. Arch. Biochem. Biophys., 365 , 223-230 (1999).

24) M. Zamocky, C. Schumann, C. Sygmund, J. O’Callaghan, A.D. Dobson, R. Ludwig, D. Haltrich and C.K. Peterbauer: Cloning, sequence analysis and heterologous expression in Pichia pastoris of a gene encoding a thermostable cellobiose dehydrogenase from Myriococcum thermophilum. Prot. Expres. Purif., 59, 258-265 (2008)

25) W. Harreither, C. Sygmund, M. Augustin, M. Narciso, M.L. Rabinovich, L. Gorton, D. Haltrich and R. Ludwig: Catalytic properties and classification of cellobiose dehydrogenases from ascomycetes. Appl. Environ. Microbiol., 77, 1804-1815 (2011).

26) K. Igarashi, M.F. Verhagen, M. Samejima, M. Schulein, K.E. Eriksson and T. Nishino: Cellobiose dehydrogenase from the fungi Phanerochaete chrysosporium and Humicola insolens. A flavohemoprotein from Humicola insolens contains 6-hydroxyFAD as the dominant active cofactor. J. Biol. Chem., 274 3338-3344 (1999).

27) K. Igarashi, I. Momohara, T. Nishino and M. Samejima: Kinetics of inter-domain electron transfer in flavocytochrome cellobiose dehydrogenase from the white-rot fungus Phanerochaete chrysosporium. Biochem. J., 365, 521-526 (2002).

28) M. Yoshida, T. Ohira, K. Igarashi, H. Nagasawa, K. Aida, B.M. Hållberg, C. Divne, T. Nishino and M. Samejima: Production and characterization of recombinant Phanerochaete chrysosporium cellobiose dehydrogenase in the methylotrophic yeast Pichia pastoris. Biosci. Biotechnol. Biochem., 65, 2050-2057 (2001).

29) L.A. Kelley and M.J. Sternberg: Protein structure prediction on the web: a case study using the Phyre server. Nat. Protoc., 4, 363-371 (2009).

30) B.M. Hållberg, G. Henriksson, G. Pettersson, A. Vasella and C. Divne: Mechanism of the reductive half-reaction in cellobiose dehydrogenase. J. Biol. Chem., 278, 7160-7166 (2003).

31) R. Kawai, M. Yoshida, T. Tani, K. Igarashi, T. Ohira, H. Nagasawa and M. Samejima: Production and characterization of recombinant Phanerochaete chrysosporium $\beta$-glucosidase in the methylotrophic yeast Pichia pastoris. Biosci. Biotechnol. Biochem., 67, 1-7 (2003).

32) K. Igarashi, M. Yoshida, H. Matsumura, N. Nakamura, H. Ohno, M. Samejima and T. Nishino: Electron transfer chain reaction of the extracellular flavocytochrome cellobiose dehydrogenase from the basidiomycete Phanerochaete chrysosporium. FEBS J., 272, 2869-2877 (2005).

33) K. Igarashi, T. Ishida, C. Hori and M. Samejima: Characterization of an endoglucanase belonging to a new subfamily of glycoside hydrolase family 45 of the basidiomycete Phanerochaete chrysosporium. Appl. Environ. Microbiol., 74, 5628-5634 (2008).

34) M.P. Weiner and G.L. Costa: Rapid PCR site-directed mutagenesis. PCR Method Appl., 4, S131-136 (1994).

35) V. Massey and S. Ghisla: Role of charge-transfer interactions in flavoprotein catalysis. Annal New York Acad. Sci., 227, 446-465 (2006). 